\title{
Niobium titanium nitride-based superconductor-insulator-superconductor mixers for low-noise terahertz receivers
}

\author{
B. D. Jackson ${ }^{\text {a) }}$ and G. de Lange \\ SRON Netherlands Institute for Space Research, Landleven 12, 9747 AD Groningen, The Netherlands
}

\begin{abstract}
T. Zijlstra, M. Kroug, and T. M. Klapwijk
Kavli Institute for Nanoscience, Faculty of Applied Sciences, Delft University of Technology, Lorentzweg 1, 2628 CJ Delft, The Netherlands

J. A. Stern

Jet Propulsion Laboratory, California Institute of Technology, 4800 Oak Grove Drive, Pasadena, California 91109
\end{abstract}

(Received 18 November 2004; accepted 7 April 2005; published online 27 May 2005)

\begin{abstract}
Integrating NbTiN-based microstrip tuning circuits with traditional $\mathrm{Nb}$ superconductorinsulator-superconductor (SIS) junctions enables the low-noise operation regime of SIS mixers to be extended from below 0.7 to $1.15 \mathrm{THz}$. In particular, mixers incorporating a $\mathrm{NbTiN} / \mathrm{SiO}_{2} / \mathrm{NbTiN}$ microstrip tuning circuit offer low-noise performance below $0.8-0.85 \mathrm{THz}$, although their sensitivities drop significantly at higher frequencies. Furthermore, a microstrip geometry in which $\mathrm{NbTiN}$ is used as the ground plane material only $\left(\mathrm{NbTiN} / \mathrm{SiO}_{2} / \mathrm{Al}\right)$ yields significant improvements in the sensitivities of SIS mixers operating up to $1.15 \mathrm{THz}$, with an upper operating frequency that depends upon the quality of the NbTiN layer, and thus its deposition process. Films deposited at room temperature have $T_{c}=14.4 \mathrm{~K}$ and $\rho_{n, 20 \mathrm{~K}} \sim 60 \mu \Omega \mathrm{cm}$, and offer low-noise performance up to $1 \mathrm{THz}$, whereas films deposited at $400{ }^{\circ} \mathrm{C}$ have $T_{c}=16 \mathrm{~K}$ and $\rho_{n, 20 \mathrm{~K}} \sim 110 \mu \Omega \mathrm{cm}$, and offer low-noise performance up to $1.15 \mathrm{THz}$. Taken together, these results demonstrate that the high-frequency surface resistance of a NbTiN layer depends upon the film's structural properties. Most significantly, the drop in performance that is seen at $F>1 \mathrm{THz}$ in mixers incorporating NbTiN ground planes deposited at room temperature is attributed to nonhomogeneities in the structural and electrical properties of these films, as is the poor performance of mixers that incorporate NbTiN wiring layers at $F>0.85 \mathrm{THz}$. The development of these NbTiN-based microstrip tuning circuits will enable the production of low-noise SIS mixers for the 0.8-0.96- and 0.96-1.12-THz frequency bands of the Heterodyne Instrument for the Far Infrared on board the European Space Agency's Herschel Space Observatory. (C) 2005 American Institute of Physics. [DOI: 10.1063/1.1927281]
\end{abstract}

\section{INTRODUCTION}

Low-noise, broadband terahertz mixers will enable the Heterodyne Instrument for the Far Infrared (HIFI) on board the European Space Agency's Herschel Space Observatory to provide full coverage of the $0.48-1.25-$ and $1.41-1.91-\mathrm{THz}$ spectral ranges with high sensitivities. ${ }^{1}$ Combined with the absence of absorption by atmospheric water vapor (the atmosphere is virtually opaque over the majority of this spectral range), this broad spectral coverage and high sensitivity will provide astronomers with a unique opportunity to observe spectral lines of a range of chemically and physically important molecules, atoms, and ions. However, in order to realize this potential, it has been necessary to develop superconductor-insulator-superconductor (SIS) mixer technologies to extend the low-noise performance of Nb-based SIS mixers from millimeter and low submillimeter frequencies $(F<0.7 \mathrm{THz})$ (Ref. 2) to as high as $1.25 \mathrm{THz}$.

Past work has shown that a drop in the sensitivities of traditional $\mathrm{Nb}$ SIS mixers at high frequencies ${ }^{3}$ can be largely attributed to increasing losses in Nb-based microstrip tuning

\footnotetext{
a) Also at the Kavli Institute of Nanoscience, Faculty of Applied Sciences, Delft University of Technology, Lorentzweg 1, 2628 CJ Delft, The Netherlands; electronic mail: b.d.jackson@sron.rug.nl
}

circuits at $F>F_{\text {gap }}=2 \Delta / h \sim 0.7 \mathrm{THz},{ }^{4}$, where $2 \Delta$ is the superconductor's energy gap and $F_{\text {gap }}$ is termed the "gap frequency" of the material. This drop in sensitivity was partially addressed by the integration of Al-based microstrip tuning circuits with high current-density (high- $J_{c}$ ) $\mathrm{Nb} / \mathrm{Al}-\mathrm{AlO}_{x} / \mathrm{Nb}$ SIS junctions, which yielded a double sideband receiver noise temperature of $T_{\mathrm{N}, \mathrm{rec}}=840 \mathrm{~K}$ at $1042 \mathrm{GHz} .{ }^{5}$ However, it was concluded that the ultimate performance of these mixers was limited by a combination of shot noise in their high current-density $\mathrm{AlO}_{x}$-based tunnel junctions and $\sim 5-6 \mathrm{~dB}$ of coupling losses in their Al-based tuning circuits. ${ }^{6}$ Thus, one of the goals in the development of low-noise terahertz SIS mixers has been the development of microstrip tuning circuits based upon superconductors with higher superconducting transition temperatures than the $T_{c}=9.2 \mathrm{~K}$ that is typical for $\mathrm{Nb}$.

The first candidate to fill this role was $\mathrm{NbN}$, as its $T_{c}$ $\sim 16 \mathrm{~K}$ was expected to offer low-loss performance below its superconducting gap frequency $F_{\text {gap }}=2 \Delta / h \sim 1.2 \mathrm{THz}$, where $\Delta_{\mathrm{NbN}}=(3.56-4.16) k_{\mathrm{B}} T_{c, \mathrm{NbN}}$ is taken from Ref. 7 . However, the development of NbN-based SIS mixers had only limited success, partly due to the excess shot noise that is observed in some NbN SIS junctions, ${ }^{8}$ and partly due to the anomalously high surface resistance of $\mathrm{NbN}$ films depos- 
ited at room temperature on silicon or fused quartz. ${ }^{9}$ In contrast, NbTiN-based SIS mixers have yielded record lownoise receiver sensitivities at frequencies between 0.75 and $1 \mathrm{THz}^{10-12}$ (Note that recent results have demonstrated a significant improvement in the performance of $\mathrm{NbN}$-based SIS mixers, ${ }^{13}$ with a mixer incorporating a $\mathrm{NbN} / \mathrm{SiO}_{2} / \mathrm{Al}$ tuning circuit yielding a sensitivity at $0.8 \mathrm{THz}$ that is similar to those demonstrated previously in NbTiN-based mixers.)

This paper summarizes the design and performance of two waveguide SIS mixers incorporating NbTiN-based microstrip tuning circuits: one with a $\mathrm{NbTiN} / \mathrm{SiO}_{2} / \mathrm{NbTiN}$ microstrip in which the NbTiN layers are deposited at room temperature and one with a $\mathrm{NbTiN} / \mathrm{SiO}_{2} / \mathrm{Al}$ microstrip in which the NbTiN ground plane is deposited at $400{ }^{\circ} \mathrm{C}$. Together with previously reported results for quasioptical ${ }^{11}$ and waveguide ${ }^{12}$ mixers containing $\mathrm{NbTiN} / \mathrm{SiO}_{2} / \mathrm{Al}$ tuning circuits in which the NbTiN ground plane is deposited at room temperature, these results demonstrate that the use of NbTiNbased tuning circuits enables the production of low-noise SIS receivers for frequencies between 0.7 and $1.15 \mathrm{THz}$. These results are also compared with knowledge of the electrical and structural properties of $\mathrm{NbTiN}$ and $\mathrm{NbN}$ films deposited at different substrate temperatures and on different substrate materials to yield qualitative conclusions about the highfrequency surface resistances of the $\mathrm{NbTiN}$ films that are used here.

\section{MIXER DESIGN AND SIS DEVICE FABRICATION}

As is summarized in Table I, the results are presented and discussed here for four basic mixer geometries that com- bine different $\mathrm{rf}$ geometries and microstrip tuning circuit material combinations. Of the four mixers, three are waveguide mixers-mixers A and B, and that described in Ref. 12. The fourth mixer is the quasioptical mixer described in Ref. 11. Mixer A incorporates a full-height $1-\mathrm{THz}$ waveguide geometry that is identical to that used in Ref. 12 , while mixer B incorporates a half-height $1.04-\mathrm{THz}$ waveguide geometry that is based upon that of a $650-\mathrm{GHz}$ mixer that was developed for the James Clerk Maxwell Telescope. ${ }^{14}$ (As is discussed in detail in Ref. 15 , the $1.04-\mathrm{THz}$ mixer design is a scaled version of the $650-\mathrm{GHz}$ design, but with the substrate suspended in the substrate channel to reduce the magnitude and reactance of the waveguide probe impedance.)

Waveguide mixers A and B described here make use of twin-junction tuning circuits that are similar to that used in Ref. 12 (see Fig. 1), but with their dimensions optimized to accommodate different tuning circuit material combinations; mixer $\mathrm{A}$ includes a $\mathrm{NbTiN} / \mathrm{SiO}_{2} / \mathrm{NbTiN}$ tuning circuit in which the $\mathrm{NbTiN}$ layers are deposited at room temperature, while mixer $\mathrm{B}$ includes a $\mathrm{NbTiN} / \mathrm{SiO}_{2} / \mathrm{Al}$ microstrip tuning circuit in which the NbTiN ground plane is deposited at NASA's Jet Propulsion Laboratory at $400{ }^{\circ} \mathrm{C}$ on an $\mathrm{AlN}$ buffer layer. In comparison, the previously reported waveguide $^{12}$ and quasioptical ${ }^{11}$ mixers incorporated $\mathrm{NbTiN} / \mathrm{SiO}_{2} / \mathrm{Al}$ tuning circuits in which the ground planes were deposited at room temperature. As described in Ref. 16, the $\mathrm{NbTiN}$ films deposited at room temperature have $T_{c}$ $=14.4 \mathrm{~K}$ and $\rho_{n, 20 \mathrm{~K}}=110 \mu \Omega \mathrm{cm}$, with moderate amounts of compressive stress. In contrast, deposition at $400{ }^{\circ} \mathrm{C}$ on an

TABLE I. Mixer embedding geometries, SIS device layer parameters, and SIS tuning circuit geometries for the mixers described here, including the deposition parameters used for their NbTiN ground planes.

\begin{tabular}{|c|c|c|c|c|}
\hline & Mixer A & Mixer B & Ref. 11 & Ref. 12 \\
\hline $\begin{array}{c}\text { Mixer } \\
\text { embedding } \\
\text { geometry }\end{array}$ & $\begin{array}{l}1-\mathrm{THz} \text { full-height } \\
\text { waveguide with diagonal } \\
\text { horn }\end{array}$ & $\begin{array}{l}\text { 1.04-THz half-height } \\
\text { waveguide with } \\
\text { corrugated horn }\end{array}$ & $\begin{array}{l}\text { 0.95-THz twin-slot } \\
\text { planar antenna with } \\
\text { elliptical silicon lens }\end{array}$ & $\begin{array}{c}1-\mathrm{THz} \text { full-height } \\
\text { waveguide with diagonal } \\
\text { horn }\end{array}$ \\
\hline Substrate & fused quartz & fused quartz & silicon & fused quartz \\
\hline Ground plane & $\begin{array}{c}300-\mathrm{nm} \mathrm{NbTiN} \\
20{ }^{\circ} \mathrm{C}, \mathrm{Al}_{2} \mathrm{O}_{3} \text { buffer } \\
T_{c}=14.4 \mathrm{~K} \\
\rho_{n, 20 \mathrm{~K}}=110 \mu \Omega \mathrm{cm}\end{array}$ & $\begin{array}{c}300-\mathrm{nm} \text { NbTiN } \\
400{ }^{\circ} \mathrm{C}, \mathrm{AlN} \text { buffer } \\
T_{c}=16 \mathrm{~K} \\
\rho_{n, 20 \mathrm{~K}}=60 \mu \Omega \mathrm{cm}\end{array}$ & $\begin{array}{c}300-\mathrm{nm} \mathrm{NbTiN} \\
20^{\circ} \mathrm{C}, \mathrm{Al}_{2} \mathrm{O}_{3} \text { buffer } \\
T_{c}=14.4 \mathrm{~K} \\
\rho_{n, 20 \mathrm{~K}}=110 \mu \Omega \mathrm{cm}\end{array}$ & $\begin{array}{c}300-\mathrm{nm} \mathrm{NbTiN} \\
20{ }^{\circ} \mathrm{C}, \mathrm{Al}_{2} \mathrm{O}_{3} \text { buffer } \\
T_{c}=14.4 \mathrm{~K} \\
\rho_{n, 20 \mathrm{~K}}=110 \mu \Omega \mathrm{cm}\end{array}$ \\
\hline $\begin{array}{l}\text { Dielectric } \\
\text { layer }\end{array}$ & $\begin{array}{c}250-\mathrm{nm} \mathrm{SiO}{ }_{2} \\
\varepsilon_{r}=3.8 \text { (assumed) }\end{array}$ & $\begin{array}{c}250-\mathrm{nm} \mathrm{SiO}_{2} \\
\varepsilon_{r}=3.8 \text { (assumed) }\end{array}$ & $\begin{array}{c}250-\mathrm{nm} \mathrm{SiO}_{2} \\
\varepsilon_{r}=3.8 \text { (assumed) }\end{array}$ & $\begin{array}{c}250-\mathrm{nm} \mathrm{SiO}_{2} \\
\varepsilon_{r}=3.8 \text { (assumed) }\end{array}$ \\
\hline Wiring layer & $\begin{array}{c}400-\mathrm{nm} \mathrm{NbTiN} \\
T_{c}=14.4 \mathrm{~K} \\
\rho_{n, 20 \mathrm{~K}}=110 \mu \Omega \mathrm{cm}\end{array}$ & $\begin{array}{c}\quad 400-\mathrm{nm} \mathrm{Al} \\
\sigma_{4 \mathrm{~K}} \sim 2.5 \times 10^{8} \Omega^{-1} \mathrm{~m}^{-1}\end{array}$ & $\begin{array}{c}400-\mathrm{nm} \mathrm{Al} \\
\sigma_{4 \mathrm{~K}} \sim 2.5 \times 10^{8} \Omega^{-1} \mathrm{~m}^{-1}\end{array}$ & $\begin{array}{c}400-\mathrm{nm} \mathrm{Al} \\
(+100-\mathrm{nm} \mathrm{Nb}) \\
\sigma_{4 \mathrm{~K}} \sim 2.5 \times 10^{8} \Omega^{-1} \mathrm{~m}^{-1}\end{array}$ \\
\hline SIS junction & $\begin{array}{c}\mathrm{Nb} / \mathrm{Al}-\mathrm{AlO}_{x} / \mathrm{Nb} \\
J_{c}=7-8 \mathrm{kA} / \mathrm{cm}^{2} \\
A=1 \mu \mathrm{m}^{2} \\
R_{2.0 \mathrm{mV}} / R_{\mathrm{N}} \sim 10\end{array}$ & $\begin{array}{c}\mathrm{Nb} / \mathrm{Al}-\mathrm{AlO}_{x} / \mathrm{Nb} \\
J_{c}=6-7 \mathrm{kA} / \mathrm{cm}^{2} \\
A=1-1.2 \mu \mathrm{m}^{2} \\
R_{2.0 \mathrm{mV}} / R_{\mathrm{N}} \sim 30-50\end{array}$ & $\begin{array}{c}\mathrm{Nb} / \mathrm{Al}-\mathrm{AlO}_{x} / \mathrm{Nb} \\
J_{c}=7-8 \mathrm{kA} / \mathrm{cm}^{2} \\
A=0.7-1.05 \mu \mathrm{m}^{2} \\
R_{2.0 \mathrm{mV}} / R_{\mathrm{N}}=30-50\end{array}$ & $\begin{array}{c}\mathrm{Nb} / \mathrm{Al}-\mathrm{AlO}_{x} / \mathrm{Nb} \\
J_{c}=7-8 \mathrm{kA} / \mathrm{cm}^{2} \\
A=0.58-0.67 \mu \mathrm{m}^{2} \\
R_{2.0 \mathrm{mV}} / R_{\mathrm{N}}=10-17\end{array}$ \\
\hline $\begin{array}{l}\text { Transformer } \\
\text { dimensions }\end{array}$ & $\begin{array}{c}W_{\mathrm{tr}}=6 \mu \mathrm{m} \\
L_{\mathrm{tr}}=20-26 \mu \mathrm{m}\end{array}$ & $\begin{array}{c}W_{\mathrm{tr}}=4-5 \mu \mathrm{m} \\
L_{\mathrm{tr}}=20-22 \mu \mathrm{m}\end{array}$ & $\begin{array}{l}W_{\mathrm{tr}}=5 \mu \mathrm{m} \\
L_{\mathrm{tr}}=23 \mu \mathrm{m}\end{array}$ & $\begin{array}{c}W_{\mathrm{tr}}=7 \mu \mathrm{m} \\
L_{\mathrm{tr}}=18-23 \mu \mathrm{m}\end{array}$ \\
\hline $\begin{array}{l}\text { Junction } \\
\text { separation }\end{array}$ & $\begin{array}{c}W_{s}=10 \mu \mathrm{m} \\
L_{s}=4-7 \mu \mathrm{m}\end{array}$ & $\begin{array}{c}W_{s}=6-7 \mu \mathrm{m} \\
L_{s}=3.5-5.5 \mu \mathrm{m}\end{array}$ & $\begin{array}{l}W_{s}=5 \mu \mathrm{m} \\
L_{s}=4 \mu \mathrm{m}\end{array}$ & $\begin{array}{c}W_{s}=11 \mu \mathrm{m} \\
L_{s}=4-7 \mu \mathrm{m}\end{array}$ \\
\hline
\end{tabular}



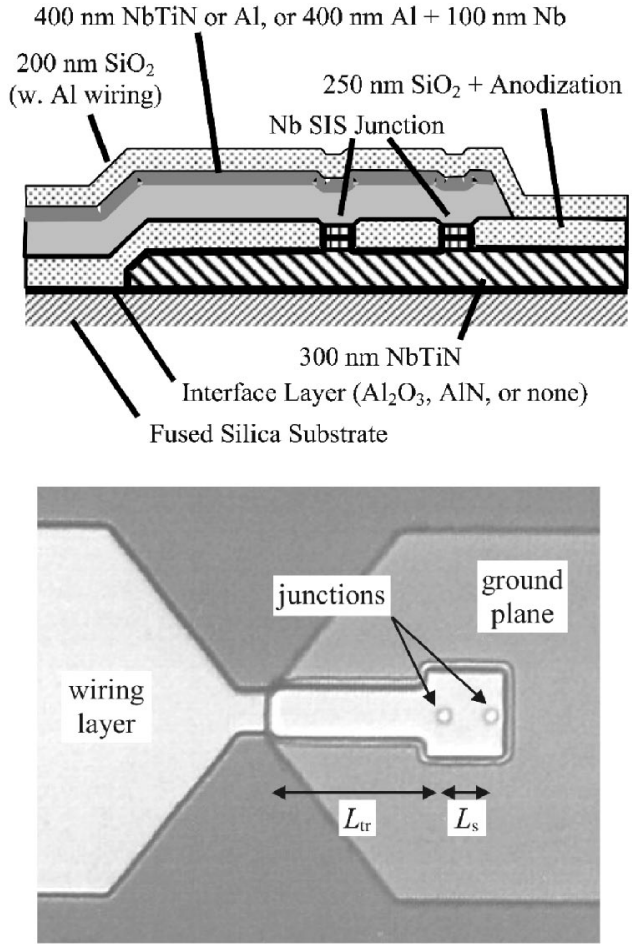

FIG. 1. Twin-junction tuning circuit geometry. (top) A cross section of the microstrip geometry. (bottom) A photograph of a 1-THz SIS device incorporating two $1-\mu \mathrm{m}^{2}$ SIS junctions and a $\mathrm{NbTiN} / \mathrm{SiO}_{2} / \mathrm{Al}$ microstrip (modified from Ref. 12). All of the waveguide devices presented here have this basic geometry, but with lengths and widths of the transformer and the junction separation (identified by $L_{\mathrm{tr}}$ and $L_{s}$ ) that are optimized to suit the surface impedances of the microstrip's ground plane and wiring layer (see Table I). As is described in Ref. 11, the quasioptical devices presented here also include a two-junction tuning circuit.

AlN buffer layer yields NbTiN ground planes with $T_{c}$ $=16 \mathrm{~K}$ and $\rho_{n, 20 \mathrm{~K}}=60 \mu \Omega \mathrm{cm}$ (also with moderate amounts of compressive stress). ${ }^{17}$

In all cases, the mixers described here include $\mathrm{Nb} / \mathrm{Al}-\mathrm{AlO}_{x} / \mathrm{Nb}$ SIS junctions with areas of $0.6-1.2 \mu \mathrm{m}^{2}$ and current densities ranging between 6 and $8 \mathrm{kA} / \mathrm{cm}^{2}$. The junctions were defined by contact optical lithography and a combination of reactive ion etching of the two $\mathrm{Nb}$ electrodes and $\mathrm{Ar}$ sputter etching of the $\mathrm{Al}-\mathrm{AlO}_{x}$ barrier layer. In the devices in mixer $\mathrm{A}$ and in the previously reported results, the $\mathrm{Nb}$ layers were etched in a $\mathrm{CF}_{4}+\mathrm{O}_{2}$ plasma, while in the devices in mixer $\mathrm{B}$ the $\mathrm{Nb}$ layers were etched in a $\mathrm{SF}_{6}$-based plasma to improve the anisotropy of the etch (and thus, the control of the junction size). In addition, the junction etch in the devices in mixer $\mathrm{B}$ included an additional $\mathrm{O}_{2}$ reactive ion etch of the resist pattern following the etch of the top $\mathrm{Nb}$ electrode and prior to sputter etching the $\mathrm{Al}-\mathrm{AlO}_{x}$ barrier. Hereafter referred to as a "resist-recessing" step, this oxygen etch shrinks the resist pattern so that the reactive ion etch of the bottom $\mathrm{Nb}$ electrode also etches the exposed edges of the top $\mathrm{Nb}$ electrode. The end result is thus a stepped junction profile in which the size of the top $\mathrm{Nb}$ electrode, which defines the active area of the $\mathrm{Al}-\mathrm{AlO}_{x}$ tunnel barrier, is defined by the resist pattern after the $\mathrm{O}_{2}$ plasma etch, while the size of the bottom $\mathrm{Nb}$ electrode is defined by the resist size before the $\mathrm{O}_{2}$ etch. This resist-recessing step has been introduced because it ensures that the edges of the $\mathrm{Al}-\mathrm{AlO}_{x}$ tunnel bar- rier are not exposed to the Ar sputter etch of the barrier, as the final junction size is only reached at the completion of the second $\mathrm{SF}_{6}$-based reactive ion etch. This, in turn, is expected to improve the quality of the junctions by reducing the risk of damage to the edges of the tunnel barrier. Note that the term "junction quality" is defined here as the junction's subgap to normal-state resistance ratio $R_{2.0 \mathrm{mV}} / R_{\mathrm{N}}$, such that high-quality junctions are those with low subgap leakage currents.

Other than the junction definition step (and the material of their wiring layers, which must be patterned in different ways), the other significant difference between the processes used for the devices in mixers A and B is that the use of an elevated-temperature deposition process for the ground planes in mixer B requires that the ground plane be patterned by reactive ion etching instead of lift-off. The sharp edge that this etch produces along the perimeter of the ground plane pattern can lead to problems with step coverage, as evidenced by short circuits between the wiring layer and the ground plane. This problem has been partially mitigated by "planarizing" the wafer after the NbTiN etch with a $\mathrm{SiO}_{2}$ layer of approximately the same thickness as the NbTiN ground plane that is patterned to form the mirror image of the ground plane. However, this solution is not ideal, the buildup of the three $\mathrm{SiO}_{2}$ layers in the devices (this planarization layer, the dielectric layer in the microstrip tuning circuit, and a passivation layer that protects the $\mathrm{Al}$ wiring layer from chemical attack) can lead to adhesion problems that reduce the process yield.

In all cases, the $\mathrm{SiO}_{2}$ layers are deposited by rf magnetron sputtering of a $\mathrm{SiO}_{2}$ target, while the $\mathrm{Al}$ and $\mathrm{Nb}$ layers are deposited by dc magnetron sputtering. A combination of a reasonable vacuum quality $\left(P_{\text {background }}<5 \times 10^{-7}\right.$ mbars $)$ and a relatively high deposition rate $(>130 \mathrm{~nm} / \mathrm{min})$ yields 400-nm-thick aluminum wiring layers with a typical "residual resistance ratio" of $R R R=\left(R_{300 \mathrm{~K}}-R_{4 \mathrm{~K}}\right) / R_{4 \mathrm{~K}} \geqslant 10$ at $4 \mathrm{~K}$ (yielding $\sigma_{\mathrm{Al}, 4 \mathrm{~K}}>3 \times 10^{8} \Omega^{-1} \mathrm{~m}^{-1}$. Note that $\sigma_{\mathrm{Al}, 4 \mathrm{~K}}$ $\sim(2-2.5) \times 10^{8} \Omega^{-1} \mathrm{~m}^{-1}$ is typically used when modeling the performance of these devices, to account for the fact that the $\mathrm{rf}$ radiation in the microstrip probes the bottom part of the Al layer, which is likely to have a local resistivity that is slightly higher than that of the bulk of the film. This is because the $\mathrm{SiO}_{2}$ dielectric layer is not expected to be a good substrate for the initial growth of the Al film. The device performance is not expected to be strongly dependent upon the resistivity of the $\mathrm{Al}$ layer.

\section{CURRENT-VOLTAGE CHARACTERISTICS}

Figure 2 presents typical low-temperature currentvoltage characteristics for SIS junctions from mixers A and $\mathrm{B}$, with $\mathrm{NbTiN} / \mathrm{SiO}_{2} / \mathrm{NbTiN}$ and $\mathrm{NbTiN} / \mathrm{SiO}_{2} / \mathrm{Al}$ microstrip tuning circuits, respectively. As seen in Refs. 11 and 12, the use of a $\mathrm{NbTiN} / \mathrm{SiO}_{2} / \mathrm{Al}$ tuning circuit in mixer $\mathrm{B}$ yields a current-voltage characteristic that is typical of a Nb SIS junction, with a gap voltage of $V_{\text {gap }}=2.7-2.8 \mathrm{mV}$. When compared to the previous results of waveguide mixers in Ref. 12, the qualities of the junctions in mixer B (as measured by their subgap to normal-state resistance ratios) are 

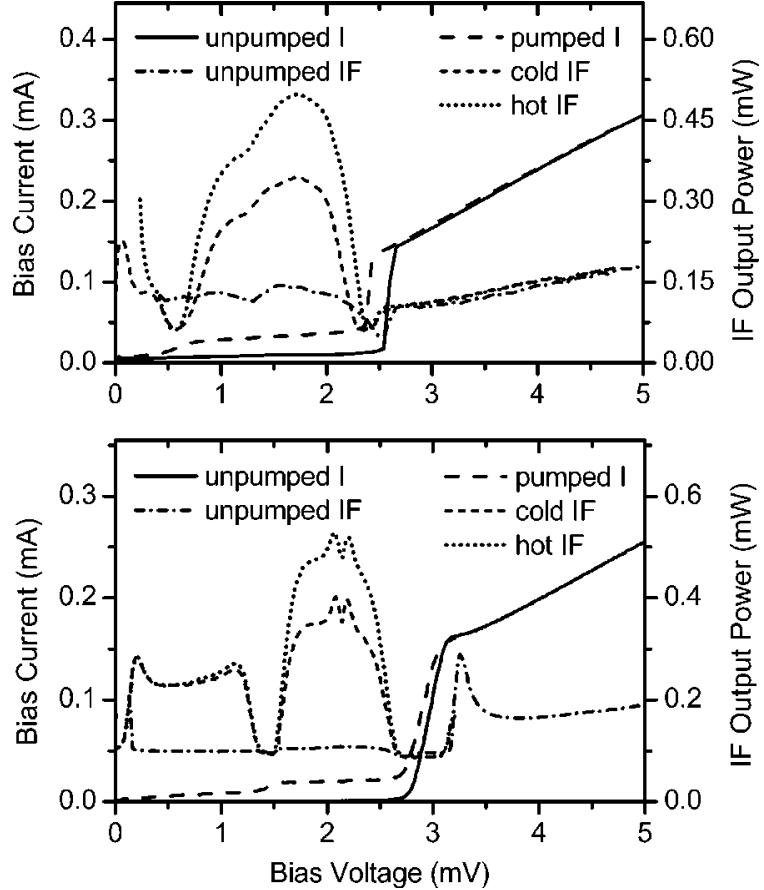

FIG. 2. Bias current and intermediate frequency (IF) output power as a function of bias voltage for mixer A (top, with a $\mathrm{NbTiN} / \mathrm{SiO}_{2} / \mathrm{NbTiN}$ tuning circuit) and mixer $\mathrm{B}$ (bottom, with a $\mathrm{NbTiN} / \mathrm{SiO}_{2} / \mathrm{Al}$ tuning circuit). The current-voltage characteristic of mixer B is typical for a Nb SIS junction (with $\sim 1 \Omega$ of series resistance in the $\mathrm{Al}$ wiring layer). However, that of mixer A shows evidence of gap voltage suppression due to the trapping of heat in the $\mathrm{Nb}$ junction electrodes, as demonstrated by the suppression of its gap voltage by the application of LO pumping power.

excellent, with $R_{2.0 \mathrm{mV}} / R_{\mathrm{N}} \sim 30-50$ for this junction with $J_{c}=6.5 \mathrm{kA} / \mathrm{cm}^{2}$. This high quality has been repeated in several batches of devices produced using the resist-recessing step during the junction definition process and is taken as evidence that the incorporation of this extra process step is having a positive impact on junction quality. Note that previous work also yielded high junction qualities, including $R_{2.0 \mathrm{mV}} / R_{\mathrm{N}}$ as high as 60 in the batch of quasioptical mixers described in Ref. 11. However, previously characterized waveguide mixers were typically characterized by poorer junction qualities, such as the value of $R_{2.0 \mathrm{mV}} / R_{\mathrm{N}}=10$ seen in Ref. 12.

In contrast with the results of mixer $\mathrm{B}$, the currentvoltage characteristic of mixer A, with an all-NbTiN tuning circuit, is characterized by a suppressed gap voltage of $V_{\text {gap }}$ $\sim 2.6 \mathrm{mV}$. Additionally, although not shown here, the current-voltage characteristic of mixer A is characterized by hysteresis at the gap voltage, with $V_{\text {gap }} \approx 2.65 \mathrm{mV}$ if the gap is approached from the subgap region, and $V_{\text {gap }} \approx 2.55 \mathrm{mV}$ if the gap is approached from the normal-state region. As is discussed in Ref. 18, this gap voltage suppression is attributed to heat being trapped in the junction electrodes due to the fact that they are flanked by NbTiN layers that have larger superconducting energy gaps than that of $\mathrm{Nb}$. As is also seen in Fig. 2, this heat trapping results in a further suppression of the gap voltage when local oscillator power is introduced. The net effect of this gap voltage suppression is to significantly reduce the bias voltage range that is available for mixer operation, which could effectively limit the maxi- mum operating frequency of this mixer geometry- $\mathrm{Nb}$ SIS junctions combined with an all-NbTiN microstrip.

\section{RF MEASUREMENT SETUP}

All receiver sensitivities reported here are double-side band receiver noise temperatures determined from $Y$-factor measurements with a room-temperature "hot" blackbody and a 77-K "cold" blackbody. The effective input power levels from these loads, plus the noise contributions from optical losses, are determined from the Callen-Welton formulation for the thermal radiation of a blackbody. ${ }^{19}$

The heterodyne mixer measurements reported here were performed in two test cryostat configurations. Backward wave oscillators and carcinotron local oscillators (LO's) combine to provide coverage of a large fraction of the $0.75-1.15-\mathrm{THz}$ range. In both test configurations, the mixer is mounted on the helium-cooled 4-K stage of a HD8 Infrared Labs cryostat, with the $\mathrm{LO}$ and signal beams being combined with a dielectric beamsplitter and coupled into the cryostat via an optical window and infrared heat filters. The intermediate frequency (IF) output from the mixer is coupled to a cryogenic low-noise amplifier via an isolator that suppresses the formation of standing waves between the mixer and the amplifier. After cryogenic amplification, the mixer's IF output signal is coupled out of the cryostat, further amplified, filtered, and then detected with a power meter. Unless specifically stated otherwise, the results presented here are obtained with the mixer at an operating temperature of $2-3 \mathrm{~K}$. (This is achieved by pumping on the cryostat's helium bath.) The improvement in the receiver noise temperature that is obtained by reducing the operating temperature from $4.5 \mathrm{~K}$ to less than $3 \mathrm{~K}$ is typically $25 \%-30 \%$.

As is summarized in Table II, the primary differences between the measurement configurations used for the four sets of results considered here are the IF band and the optical elements in the path between the mixer and the hot/cold load. In particular, mixer A and those in Refs. 11 and 12 were tested at $F_{\mathrm{IF}}=1.5 \mathrm{GHz}$ with a conventional hot/cold configuration that included a beamsplitter for LO injection located between the hot/cold load and the cryostat, a dielectric film as a vacuum window, and Zitex G104 sheets as infrared heat filters. In contrast, mixer B was tested at $F_{\mathrm{IF}}=4-8 \mathrm{GHz}$ with a vacuum hot/cold system in which the beamsplitter is located inside the vacuum, which allows the vacuum window to be removed from the signal path. Additionally, while early tests of mixer A and the mixer described in Ref. 12 made use of a dielectric lens to focus the incident optical beam into the mixer's horn antenna, mixer B was tested with a cold mirror as a focusing element, to further reduce the optical losses in the system. A focusing element was not needed in testing the quasioptical mixer reported in Ref. 11 because the elliptical lens used in that mixer produces a relatively collimated rf beam.

Note that the use of a broadband, higher-frequency IF system for testing mixer B increases the IF system's noise contribution to the total receiver noise slightly, since the input noise temperature of the $4-8-\mathrm{GHz}$ IF system is $T_{\mathrm{N}, \mathrm{IF}}$ $=8-10 \mathrm{~K}$, while the $1.5-\mathrm{GHz}$ IF system is characterized by 
TABLE II. Receiver optics, intermediate frequency (IF) system configurations, and mixer operating temperatures used for testing mixers A and B and in Refs. 11 and 12

\begin{tabular}{|c|c|c|c|c|}
\hline & Mixer A & Mixer B & Ref. 11 & Ref. 12 \\
\hline Beamsplitter (BS) & 14-, 49- $\mu \mathrm{m}$ Mylar & 14-, 49- $\mu \mathrm{m}$ Mylar & $\begin{array}{c}\text { 6-, 14-, } 49-\mu \mathrm{m} \\
\text { Mylar }\end{array}$ & 14-, 49- $\mu$ m Mylar \\
\hline Dewar window & 100- $\mu \mathrm{m}$ Mylar & not in signal path & 12- $\mu \mathrm{m}$ Kapton & 100- $\mu \mathrm{m}$ Mylar \\
\hline $\begin{array}{c}\text { Infrared } \\
\text { heat filter(s) }\end{array}$ & $\begin{array}{l}\text { Zitex G104 } \\
\text { at } 77 \mathrm{~K}\end{array}$ & $\begin{array}{l}\text { Zitex G104 } \\
\text { at } 77 \mathrm{~K}\end{array}$ & $\begin{array}{c}\text { Zitex G104 } \\
\text { at } 77 \text { and } 4.2 \mathrm{~K}\end{array}$ & $\begin{array}{l}\text { Zitex G104 } \\
\text { at } 77 \mathrm{~K}\end{array}$ \\
\hline Focusing element & $\begin{array}{l}\text { high-density } \\
\text { polyethylene lens }\end{array}$ & Au-coated mirror & $\begin{array}{l}\text { silicon lens is } \\
\text { part of mixer }\end{array}$ & $\begin{array}{c}\text { high-density } \\
\text { polyethylene lens }\end{array}$ \\
\hline $\begin{array}{l}\text { Receiver optics } \\
\text { input noise and } \\
\text { insertion loss }\end{array}$ & $\begin{array}{c}100 \mathrm{~K}, 2.0 \mathrm{~dB} \\
\text { at } 830 \mathrm{GHz} \\
\text { with } 14-\mu \mathrm{m} \mathrm{BS}\end{array}$ & $\begin{array}{c}60 \mathrm{~K}, 1.0 \mathrm{~dB} \\
\text { at } 1040 \mathrm{GHz} \\
\text { with } 14-\mu \mathrm{m} \mathrm{BS}\end{array}$ & $\begin{array}{c}35 \mathrm{~K}, 1.2 \mathrm{~dB} \\
\text { at } 970 \mathrm{GHz} \\
\text { with } 6-\mu \mathrm{m} \mathrm{BS}\end{array}$ & $\begin{array}{c}146 \mathrm{~K}, 2.5 \mathrm{~dB} \\
\text { at } 970 \mathrm{GHz} \\
\text { with } 14-\mu \mathrm{m} \mathrm{BS}\end{array}$ \\
\hline $\begin{array}{l}\text { IF bandwidth, } \\
\text { center frequency, } \\
\text { and input noise }\end{array}$ & $\begin{array}{c}\Delta F_{\mathrm{IF}}=80 \mathrm{MHz} \\
F_{\mathrm{IF}}=1.5 \mathrm{GHz} \\
T_{\mathrm{N}, \mathrm{IF}} \sim 4-5 \mathrm{~K}\end{array}$ & $\begin{array}{c}\Delta F_{\mathrm{IF}}=4 \mathrm{GHz} \\
F_{\mathrm{IF}}=4-8 \mathrm{GHz} \\
T_{\mathrm{N}, \mathrm{IF}} \sim 8-10 \mathrm{~K}\end{array}$ & $\begin{array}{c}\Delta F_{\mathrm{IF}}=80 \mathrm{MHz} \\
F_{\mathrm{IF}}=1.5 \mathrm{GHz} \\
T_{\mathrm{N}, \mathrm{IF}} \sim 4-5 \mathrm{~K}\end{array}$ & $\begin{array}{c}\Delta F_{\mathrm{IF}}=80 \mathrm{MHz} \\
F_{\mathrm{IF}}=1.5 \mathrm{GHz} \\
T_{\mathrm{N}, \mathrm{IF}} \sim 4-5 \mathrm{~K}\end{array}$ \\
\hline $\begin{array}{l}\text { Operating } \\
\text { temperature }\end{array}$ & $T_{\text {mixer }}=4.5 \mathrm{~K}$ & $T_{\text {mixer }}=2.5 \mathrm{~K}$ & $T_{\text {mixer }}=2.5 \mathrm{~K}$ & $T_{\text {mixer }}=2.5 \mathrm{~K}$ \\
\hline
\end{tabular}

$T_{\mathrm{N}, \mathrm{IF}}=4-5 \mathrm{~K}$. Given a typical single sideband conversion gain of $-10 \mathrm{~dB}$ for the mixers described here and optics losses of $1-2 \mathrm{~dB}$, this results in $\sim 30 \mathrm{~K}$ of additional double sideband receiver noise in the measurements of mixer B relative to those of mixer A and the mixers in Refs. 11 and 12. However, this effect is much less than the differences in the optical losses in the different systems-these are summa- rized in Table II. For this reason, the results obtained with different mixers are best compared after correcting the measured receiver sensitivities for the optical losses in the receiver cryostat, which yields $T_{\mathrm{N} \text {,mixer+IF }}$.

Direct detection measurements of the mixers' response bandwidths are also presented here. These were obtained with a Fourier transform spectrometer in which a Michelson
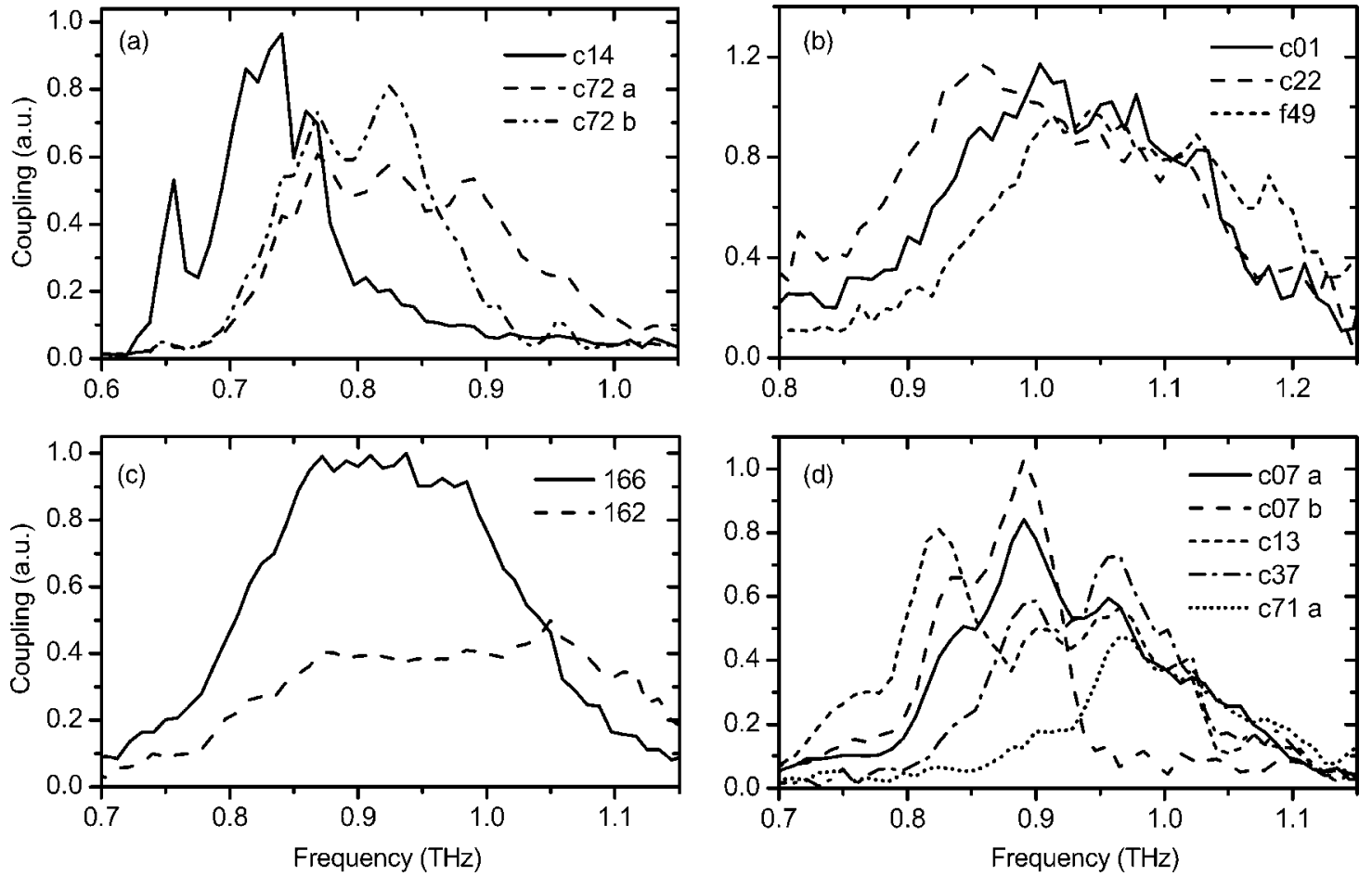

FIG. 3. Direct detection responses of several devices in: (a) mixer A with $\mathrm{NbTiN} / \mathrm{SiO}_{2} / \mathrm{NbTiN}$ tuning circuits; (b) mixer $\mathrm{B}$ with $\mathrm{NbTiN} / \mathrm{SiO}{ }_{2} / \mathrm{Al}$ tuning circuits in which the NbTiN ground plane is deposited at $400{ }^{\circ} \mathrm{C}$; (c) a quasioptical mixer with a NbTiN/SiO$/$ /Al tuning circuit in which the NbTiN is deposited at room temperature (modified from Ref. 11); and (d) a waveguide mixer with a $\mathrm{NbTiN} / \mathrm{SiO}_{2} / \mathrm{Al}$ tuning circuit in which the $\mathrm{NbTiN}$ is deposited at room temperature (modified from Ref. 12). Note that the response of device c72 in (a) is the strongest high-frequency response that has been obtained with a $\mathrm{NbTiN} / \mathrm{SiO}_{2} / \mathrm{NbTiN}$ microstrip tuning circuit, with the curves marked c72a and c72b corresponding to measurements with different backshort depths in a mixer block that includes a sliding contacting backshort. Devices with a $\mathrm{NbTiN} / \mathrm{SiO}_{2} / \mathrm{Al}$ tuning circuit in which the NbTiN ground plane is deposited at $400{ }^{\circ} \mathrm{C}$ [in (b)] offer strong coupling above $1 \mathrm{THz}$, in notable contrast with those in which the NbTiN is deposited at room temperature [in (c) and (d)]. 

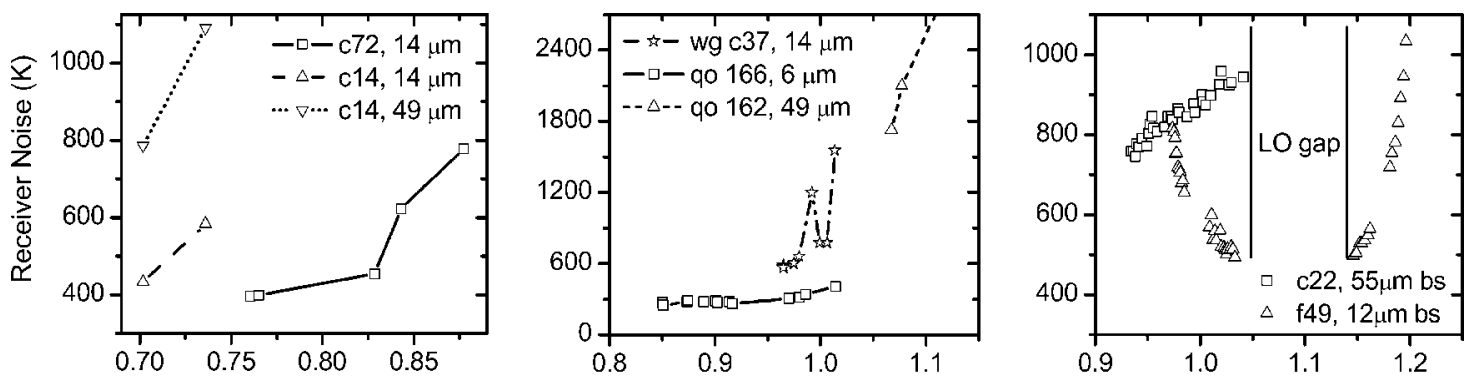

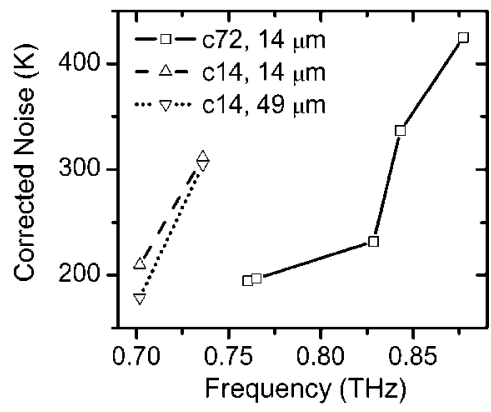

(a)

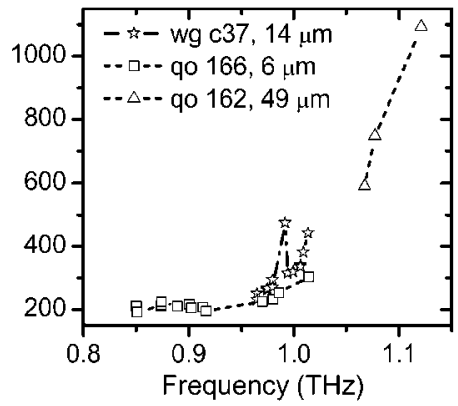

(b)

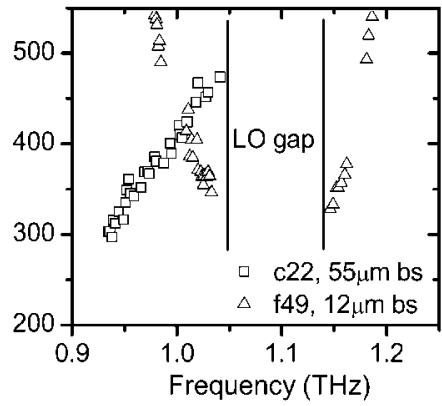

(c)

FIG. 4. Measured double sideband receiver noise temperatures of several devices in: (a) mixer A with $\mathrm{NbTiN} / \mathrm{SiO}_{2} / \mathrm{NbTiN}$ tuning circuits; (b) mixers from Refs. 11 and 12 with $\mathrm{NbTiN} / \mathrm{SiO}_{2} / \mathrm{Al}$ tuning circuits in which the $\mathrm{NbTiN}$ ground plane is deposited at room temperature; and (c) mixer B with $\mathrm{NbTiN} / \mathrm{SiO}_{2} / \mathrm{Al}$ tuning circuits in which the $\mathrm{NbTiN}$ ground plane is deposited at $400{ }^{\circ} \mathrm{C}$. All results were obtained at $T_{\text {mixer }}=2.5 \mathrm{~K}$, except for those in (a), which were obtained at $4.5 \mathrm{~K}$. All results were obtained at $F_{\mathrm{IF}}=1.5 \mathrm{GHz}$, with $T_{\mathrm{N}, \mathrm{IF}} \sim 4-5 \mathrm{~K}$, except for those in (c), which were obtained at $F_{\mathrm{IF}}$ $=4-8 \mathrm{GHz}$, with $T_{\mathrm{N}, \mathrm{IF}} \sim 8-10 \mathrm{~K}$. In addition to the individual device labels, the legends also identify the beamsplitter thickness that was used for each measurement-a thick beamsplitter was used to compensate for a lack of LO power in some measurements. Because this significantly degrades these receiver

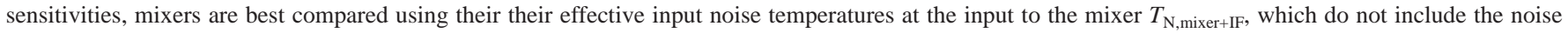
contributions of the receiver optics.

interferometer is used as a tunable signal source, the beam path of which can be evacuated to eliminate absorption lines of atmospheric water vapor from the measured spectra.

\section{RF MEASUREMENT RESULTS}

Figure 3 presents the measured direct detection spectra of several devices in each of mixers A and B, plus spectra from the mixers presented in Refs. 11 and 12. Two particularly notable features are seen in these spectra. First, it is noted that while mixer A shows strong responses at lower frequencies, none of the devices with $\mathrm{NbTiN} / \mathrm{SiO}_{2} / \mathrm{NbTiN}$ tuning circuits that were tested have strong responses above $\sim 0.85$ THz. Indeed, device c72 in Fig. 3(a) offered the best high-frequency performance of the devices tested in mixer A. However, measurements with different backshort depthsc72a and c72b in Fig. 3(a) - show that its highest sensitivities are obtained below $0.85 \mathrm{THz}$. This is in sharp contrast with the previously reported results obtained in the same mixer block using devices with $\mathrm{NbTiN} / \mathrm{SiO}_{2} / \mathrm{Al}$ tuning circuits [see Fig. 3(d) and Ref. 12], in which strong responses were seen up to $1 \mathrm{THz}$. The second point that is noted here is that mixer $\mathrm{B}$ (with a $\mathrm{NbTiN} / \mathrm{SiO}_{2} / \mathrm{Al}$ tuning circuit incorporating a $\mathrm{NbTiN}$ ground plane that was deposited at $400{ }^{\circ} \mathrm{C}$ ) yields strong responses at frequencies well above the 1 -THz upper frequency limit that was seen in the performance of the devices in Refs. 11 and 12 [see Figs. 3(b) and 3(c)], which incorporated NbTiN ground planes that were deposited at room temperature.

These observations of the frequency dependence of the mixers' direct detection responses are mirrored in their mea- sured heterodyne sensitivities, which are summarized in Fig. 4. In particular, Fig. 4(a) clearly demonstrates that mixer A, with a $\mathrm{NbTiN} / \mathrm{SiO}_{2} / \mathrm{NbTiN}$ tuning circuit, offers low-noise performance at frequencies below $0.85 \mathrm{THz}$, with the input noise temperatures of these mixers increasing sharply at higher frequencies. (As noted above, device $\mathrm{c} 72$ has the best high-frequency sensitivity of all of the devices of this type that were measured.) In contrast, as is seen in Fig. 4(b), mixers with $\mathrm{NbTiN} / \mathrm{SiO}_{2} / \mathrm{Al}$ tuning circuits in which the $\mathrm{NbTiN}$ ground plane is deposited at room temperature offer low-noise performance at frequencies up to $1 \mathrm{THz}$. However, the receiver and mixer sensitivities from Refs. 11 and 12 are characterized by a sharp increase in their noise temperatures at frequencies higher than $1 \mathrm{THz}$. Fortunately, the results seen in Fig. 4(c) clearly demonstrate that the use (in mixer B) of NbTiN films deposited at $400{ }^{\circ} \mathrm{C}$ allows this upper frequency limit to be pushed to at least $1.1-1.15 \mathrm{THz}$.

Comparing the sensitivities of these receivers and mixers, it is seen that the mixers with $\mathrm{NbTiN} / \mathrm{SiO}_{2} / \mathrm{NbTiN}$ tuning circuits yield $T_{\mathrm{N} \text {,mixer+IF }} \approx 200 \mathrm{~K}$ at $0.75 \mathrm{THz}$ (at an operating temperature of $T_{\text {mixer }}=4.5 \mathrm{~K}$ ), while mixers with a room-temperature $\mathrm{NbTiN} / \mathrm{SiO}_{2} / \mathrm{Al}$ tuning circuit yield $T_{\mathrm{N}, \text { mixer+IF }} \approx 200 \mathrm{~K}$ at $0.85-0.9 \mathrm{THz}$ (at $T_{\text {mixer }}=2.5 \mathrm{~K}$ ). Both of these results are obtained at $F_{\mathrm{IF}}=1.5 \mathrm{GHz}$, with $T_{\mathrm{N}, \mathrm{IF}}$ $=4-5 \mathrm{~K}$. Finally, the mixers with an elevated-temperature NbTiN ground plane and an $\mathrm{Al}$ wiring layer yield $T_{\mathrm{N}, \text { mixer+IF }}=300-350 \mathrm{~K}$ at $1.0-1.1 \mathrm{THz}$ and $F_{\mathrm{IF}}=4-8 \mathrm{GHz}$ (with $T_{\mathrm{N}, \mathrm{IF}}=8-10 \mathrm{~K}$ at $T_{\text {mixer }}=2.5 \mathrm{~K}$ ). 


\section{DISCUSSION}

As mentioned previously, mixers in which $\mathrm{Nb}$ SIS junctions are integrated into a $\mathrm{NbTiN} / \mathrm{SiO}_{2} / \mathrm{NbTiN}$ microstrip tuning circuit are characterized by heat trapping in the $\mathrm{Nb}$ junction electrodes. Following the discussion in Ref. 18, it is noted that this heat trapping is sufficient to raise the temperature of the junction electrodes by $\sim 1 \mathrm{~K}$ due to the power dissipated by the dc bias voltage alone, plus another $1 \mathrm{~K}$ when the junctions are pumped by local oscillator power at a level that is similar to that which is used in heterodyne mixer measurements. Operationally, one effect of this heating is to suppress the junctions' gap voltages, which could limit their upper operating frequency, if the high-frequency sensitivity of the mixers was not limited by other factors. Specifically, the observed $0.8-0.85-\mathrm{THz}$ upper frequency limit in these mixers is lower than the $0.9-1.0-\mathrm{THz}$ operating frequency limit that would be expected from their junctions' 2.4-mV gap voltages when LO power is applied. Furthermore, the junctions' gap voltages are $\sim 2.6 \mathrm{mV}$ in the absence of LO power, so an even larger upper frequency limit would be expected in their direct detection responses, which are measured without a LO.

The heating effect observed in mixer A does complicate the operation of these mixers, due the fact that the width of the first photon step below the gap voltage is dependent upon the LO pumping level. Thus, if a $\mathrm{NbTiN} / \mathrm{SiO}_{2} / \mathrm{NbTiN}$ tuning circuit was to be used at frequencies below $0.8 \mathrm{THz}$, it would be desirable to reduce this heating effect. For example, the use of a $\mathrm{Nb} / \mathrm{Al}-\mathrm{AlN}_{x} / \mathrm{NbTiN}$ junction ${ }^{20}$ would allow heat to escape into the top NbTiN wiring layer via the NbTiN junction electrode. (Note that the first photon step below the gap voltage is the region in which the mixer's optimum bias voltage is found.)

The drop in sensitivity above $\sim 0.85 \mathrm{THz}$ in mixers incorporating $\mathrm{NbTiN} / \mathrm{SiO}_{2} / \mathrm{NbTiN}$ tuning circuits is attributed to losses in the NbTiN wiring layer that are caused by a variation in the superconducting properties of the $\mathrm{NbTiN}$ as a function of the vertical position within the film. In particular, it is noted that measurements of the $T_{c}$ of NbTiN films of varying thickness showed that the $T_{c}$ of these films drops significantly for film thicknesses less than $100 \mathrm{~nm} .{ }^{21}$ Indeed, if the thickness dependence of the $T_{c}$ of $\mathrm{NbN}$ films deposited at room temperature on silicon ${ }^{7}$ is combined with $T_{c}$ $=14.4 \mathrm{~K}$ for bulk NbTiN, a value of $T_{c} \sim 11 \mathrm{~K}$ is estimated for a 25-nm-thick layer of NbTiN. (This value is consistent with the trend seen in Ref. 21 for NbTiN films.) If this value is then used as the effective $T_{c}$ of a NbTiN wiring layer, the effective gap frequency of this film is determined to be $0.8-0.9 \mathrm{THz}$ [using $F_{\text {gap,NbTiN }}=(3.56-4.16) k_{\mathrm{B}} T_{c} / h$, from previously determined relationships for similar $\mathrm{NbN}$ films ${ }^{7}$, which is consistent with the observed upper frequency limit in these devices. Note that because the observed drop in $T_{c}$ with decreasing film thickness occurs even though the thicknesses in question (tens of nanometer) are much larger than the expected coherence length for these types of films (it is $\sim 7 \mathrm{~nm}$ in single-crystal $\mathrm{NbN}$ films ${ }^{22}$ ), this drop is not attributed to the superconducting proximity effect.

The fact that the NbTiN ground plane in a
$\mathrm{NbTiN} / \mathrm{SiO}_{2} / \mathrm{Al}$ microstrip can be used to higher frequencies than the $0.85-\mathrm{THz}$ upper frequency limit seen in the performance of mixers with a NbTiN wiring layer is attributable to the fact that the fields in a microstrip are concentrated between the ground plane and wiring layer. Thus, the microstrip's performance is strongly influenced by the quality of the bottom tens of nanometer of a $\mathrm{NbTiN}$ wiring layer, which is precisely the region in which the $T_{c}$ of the film is seen to drop. In contrast, the surface resistance of a NbTiN ground plane is dominated by the bulk properties of the film, since only a small fraction of the energy in the microstrip reaches the bottom tens of nanometer of the film.

As is reported in Refs. 11 and 12, the high-frequency drop in the sensitivity of mixers with $\mathrm{NbTiN} / \mathrm{SiO}_{2} / \mathrm{Al}$ tuning circuits in which the NbTiN ground plane is deposited at room temperature is attributed to losses in the NbTiN ground plane. However, this drop is seen at $F=1 \mathrm{THz}$, instead of the $1.05-1.1 \mathrm{THz}$ that would be expected from the measured superconducting transition temperature of the film $\left(T_{c}\right.$ $=14.4 \mathrm{~K})$ and the previously observed relationship between $T_{c}$ and the superconducting energy gap in NbN films. This unexpectedly low upper frequency limit in the performance of these mixers is believed to be due to inhomongeneities in the NbTiN ground plane. In particular, the observed rf losses are attributed to the presence of grains in the NbTiN film with local $T_{c}$ 's that are lower than that which is measured for the bulk film. This argument is supported by the measurements of the crystal texture of these films, in which grains with two different crystal orientations are seen in films deposited at room temperature. ${ }^{23}$ In contrast, only one of these crystal orientations is seen in the films deposited on a latticematched $\mathrm{MgO}$ substrate, which are characterized by $T_{c}$ 's and low-temperature $\mathrm{dc}$ resistivities that are similar to those of NbTiN films deposited at $400{ }^{\circ} \mathrm{C}$ on fused quartz- $T_{c}$ $=16 \mathrm{~K}$ and $\rho_{n, 20 \mathrm{~K}}=60 \mu \Omega \mathrm{cm}$ versus the $T_{c}=14.4 \mathrm{~K}$ and $\rho_{n, 20 \mathrm{~K}}=110 \mu \Omega \mathrm{cm}$ that characterize NbTiN films deposited on fused quartz at room temperature.

The observed differences in the dc electrical and structural properties of NbTiN ground planes deposited at room temperature and $400{ }^{\circ} \mathrm{C}$ are also consistent with the observed improvement in high-frequency mixer performance that is obtained by the use of films deposited at elevated temperature. In particular, the results seen here show that the previously reported drop in mixer sensitivities at $\sim 1 \mathrm{THz}$ is pushed to at least $1.15 \mathrm{THz}$ by the use of films with $T_{c}$ $=16 \mathrm{~K}$ and $\rho_{n, 20 \mathrm{~K}}=60 \mu \Omega \mathrm{cm}$, in place of the films with $T_{c}=14.4 \mathrm{~K}$ and $\rho_{n, 20 \mathrm{~K}}=110 \mu \Omega \mathrm{cm}$ that were used previously.

\section{CONCLUSIONS}

The integration of $\mathrm{Nb} / \mathrm{Al}-\mathrm{AlO}_{x} / \mathrm{Nb}$ SIS junctions with NbTiN-based microstrip tuning circuits yields low-noise mixers in the $0.75-1.15-\mathrm{THz}$ range, with $\mathrm{NbTiN} / \mathrm{SiO}_{2}$ / NbTiN microstrip tuning circuits yielding high sensitivities below $0.8-0.85 \mathrm{THz}$, and $\mathrm{NbTiN} / \mathrm{SiO}_{2} / \mathrm{Al}$ tuning circuits yielding low-noise performance at frequencies up to $1.15 \mathrm{THz}$. The maximum frequency at which mixers incorporating $\mathrm{NbTiN} / \mathrm{SiO}_{2} / \mathrm{Al}$ tuning circuits offer high sensitivi- 
ties depends upon the conditions under which the NbTiN ground plane is deposited, with room-temperature deposition yielding high sensitivities for $F<1 \mathrm{THz}$ and deposition at $400{ }^{\circ} \mathrm{C}$ yielding high sensitivities up to $1.15 \mathrm{THz}$. These results are consistent with both the observed dc electrical characteristics of the NbTiN films (room-temperature deposition yields $T_{c}=14.4 \mathrm{~K}$ and $\rho_{n, 20 \mathrm{~K}} \sim 110 \mu \Omega \mathrm{cm}$, while deposition at $400{ }^{\circ} \mathrm{C}$ yields $T_{c}=16 \mathrm{~K}$ and $\rho_{n, 20 \mathrm{~K}} \sim 60 \mu \Omega \mathrm{cm}$ ), and previously reported measurements of the crystalline structure of NbTiN films with similar dc electrical characteristics. The observed drop in performance at $F>0.8-0.85 \mathrm{THz}$ in mixers incorporating $\mathrm{NbTiN}$ wiring layers is also consistent with the previously reported measurements of the $T_{c}$ of NbTiN layers as a function of film thickness, which indicate that the first few tens of nanometer in the film have significantly poorer electrical characteristics (especially $T_{c}$ ) than a 300 -nm-thick film.

\section{ACKNOWLEDGMENTS}

The authors would like to thank L. de Jong and W. Laauwen for performing the mixer measurements that are presented here; M. Eggens, H. Golstein, S. Kikken, D. Nguyen, C. Pieters, H. Schaeffer, H. Smit, and H. van de Stadt for their contributions to the design and construction of the mixers and test systems that were used in this work; and A. Baryshev, J.R. Gao, Th. de Graauw, N. Honingh, J. Kooi, R. LeDuc, B. Leone, S. Shitov, and J. Zmuidzinas for useful discussions. The work described in this paper was partially supported by the Technologiestichting STW (of the Netherlands), the Nederlandse Organisatie voor Wetenschappelijk Onderzoek, and the European Space Agency (via ESTEC Research Contract No. 11653/95).

${ }^{1}$ Th. de Graauw and F. P. Helmich, on behalf of the HIFI consortium, in SP-460 The Promise of the Herschel Space Observatory, edited by G. L. Pilbratt, J. Cernicharo, A. M. Heras, T. Prusti, and R. A. Harris (ESA, The Netherlands, 2001), pp. 45-52; the HIFI web-site: http://www.sron.nl/ divisions/lea/hifi

${ }^{2}$ J. W. Kooi, M. Chan, B. Bumble, H. G. LeDuc, P. Schaffer, and T. G. Phillips, Int. J. Infrared Millim. Waves 16, 2049 (1995); A. Karpov, J. Blondel, M. Voss, and K. H. Gundlach, IEEE Trans. Appl. Supercond. 9, 4456 (1999); C. E. Honingh, S. Haas, D. Hottgenroth, K. Jacobs, and J.
Stutzki, ibid. 7, 2582 (1997).

${ }^{3}$ G. de Lange, J. J. Kuipers, T. M. Klapwijk, R. A. Panhuyzen, H. van de Stadt, and M. W. M. de Graauw, J. Appl. Phys. 77, 1795 (1995).

${ }^{4}$ D. C. Mattis and J. Bardeen, Phys. Rev. 111, 412 (1958).

${ }^{5}$ M. Bin, M. C. Gaidis, J. Zmuidzinas, T. G. Phillips, and H. G. Leduc, Appl. Phys. Lett. 68, 1714 (1996).

${ }^{6}$ P. Dieleman, T. M. Klapwijk, J. R. Gao, and H. van de Stadt, IEEE Trans. Appl. Supercond. 7, 2566 (1997).

${ }^{7}$ H. Su, N. Yoshikawa, and M. Sugahara, Supercond. Sci. Technol. 9, A152 (1996); Z. Wang, A. Kawakami, Y. Uzawa, and B. Komiyama, J. Appl. Phys. 79, 7837 (1996).

${ }^{8}$ P. Dieleman, H. G. Bukkems, T. M. Klapwijk, M. Schicke, and K. H. Gundlach, Phys. Rev. Lett. 79, 3486 (1997).

${ }^{9}$ S. Kohjiro, S. Kiryu, and A. Shoji, IEEE Trans. Appl. Supercond. 3, 1765 (1993).

${ }^{10}$ J. Kawamura, J. Chen, D. Miller, J. Kooi, J. Zmuidzinas, B. Bumble, H. G. Leduc, and J. A. Stern, Appl. Phys. Lett. 75, 4013 (1999).

${ }^{11}$ B. D. Jackson, A. M. Baryshev, G. de Lange, S. V. Shitov, J.-R. Gao, N. N. Iosad, and T. M. Klapwijk, Appl. Phys. Lett. 79, 436 (2001).

${ }^{12}$ B. D. Jackson, N. N. Iosad, G. de Lange, A. M. Baryshev, W. M. Laauwen, J.-R. Gao, and T. M. Klapwijk, IEEE Trans. Appl. Supercond. 11, 653 (2001).

${ }^{13}$ A. Kawakami, Y. Uzawa, and Z. Wang, Appl. Phys. Lett. 83, 3954 (2003).

${ }^{14}$ H. van de Stadt, H. Schaeffer, and L. de Jong (unpublished); A. M. Baryshev, H. van de Stadt, H. Schaeffer, R. Hesper, T. Zijlstra, M. Zuiddam, $\mathrm{W}$. Wild, and L. de Jong, in Proceedings of the 12th International Symposium on Space THz Technology, edited by I. Mehdi (California Institute of Technology, Pasadena Hilton, Pasadena, CA, 17-19 March 1998), pp. 581-590.

${ }^{15}$ B. D. Jackson, G. de Lange, T. Zijlstra, M. Kroug, J. Kooi, J. A. Stern, and T. M. Klapwijk, IEEE Trans. Microwave Theory Tech. (submitted).

${ }^{16}$ N. N. Iosad et al., J. Appl. Phys. 88, 5756 (2000).

${ }^{17}$ J. A. Stern, B. Bumble, H. G. Leduc, J. W. Kooi, and J. Zmuidzinas, Ninth International Symposium on Space THz Technology, Pasadena, CA, 1998, edited by R. McGrath (California Institute of Technology, Humphrey's Half Moon Inn, Shelter Island, San Diego, CA, 14-16 Februrary 2001), pp. 305-313.

${ }^{18}$ B. Leone, J. R. Gao, T. M. Klapwijk, B. D. Jackson, W. M. Laauwen, and G. de Lange, Appl. Phys. Lett. 78, 1616 (2001); B. Leone, B. D. Jackson, J. R. Gao, and T. M. Klapwijk, ibid. 76, 780 (2000).

${ }^{19}$ H. B. Callen and T. A. Walton, Phys. Rev. 83, 34 (1951).

${ }^{20}$ B. Bumble, H. G. LeDuc, J. A. Stern, and K. G. Megerian, IEEE Trans. Appl. Supercond. 11, 76 (2001).

${ }^{21}$ N. N. Iosad, V. V. Roddatis, S. N. Polyakov, A. V. Varlashkin, B. D. Jackson, P. N. Dmitriev, J. R. Gao, and T. M. Klapwijk, IEEE Trans. Appl. Supercond. 11, $3832(2001)$

${ }^{22}$ A. Shoji, S. Kiryu, and S. Kohjiro, Appl. Phys. Lett. 60, 1624 (1992).

${ }^{23}$ N. N. Iosad, N. M. van der Pers, S. Grachev, V. V. Roddatis, B. D. Jackson, S. N. Polyakov, P. N. Dmitriev, and T. M. Klapwijk, J. Appl. Phys. 92, 4999 (2002) 